\title{
Valtin, Renate
}

\section{Phonologische Bewusstheit: Ein kritischer Blick auf ein modisches Konstrukt}

formal und inhaltlich überarbeitete Version der Originalveröffentlichung in:

formally and content revised edition of the original source in:

Frühe Bildung : interdisziplinäre Zeitschrift für Forschung, Ausbildung und Praxis 1 (2012) 4, S. 223-225

Bitte verwenden Sie in der Quellenangabe folgende URN oder DOI /

Please use the following URN or DOI for reference:

urn:nbn:de:0111-pedocs-209022

10.25656/01:20902

https://nbn-resolving.org/urn:nbn:de:0111-pedocs-209022

https://doi.org/10.25656/01:20902

\section{Nutzungsbedingungen}

Gewährt wird ein nicht exklusives, nicht übertragbares, persönliches und beschränktes Recht auf Nutzung dieses Dokuments. Dieses Dokument ist ausschließlich für den persönlichen, nicht-kommerziellen Gebrauch bestimmt. Die Nutzung stellt keine Übertragung des Eigentumsrechts an diesem Dokument dar und gilt vorbehaltlich der folgenden Einschränkungen Auf sämtlichen Kopien dieses Dokuments müssen alle Urheberrechtshinweise und sonstigen Hinweise auf gesetzlichen Schutz beibehalten werden. Sie dürfen dieses Dokument nicht in irgendeiner Weise abändern, noch dürfen Sie dieses Dokument für öffentliche oder kommerzielle Zwecke vervielfältigen, öffentlich ausstellen, aufführen, vertreiben oder anderweitig nutzen.

Mit der Verwendung dieses Dokuments erkennen Sie die Nutzungsbedingungen an.

\section{Terms of use}

We grant a non-exclusive, non-transferable, individual and limited right to using this document

This document is solely intended for your personal, non-commercial use. Use of this document does not include any transfer of property rights and it is conditional to the following limitations: All of the copies of this documents must retain all copyright information and other information regarding legal protection. You are not allowed to alter this document in any way, to copy it for public or commercial purposes, to exhibit the document in public, to perform, distribute or otherwise use the document in public.

By using this particular document, you accept the above-stated conditions of use.

\section{Kontakt / Contact:}

\section{peDOCS}

DIPF | Leibniz-Institut für Bildungsforschung und Bildungsinformation Informationszentrum (IZ) Bildung

E-Mail: pedocs@dipf.de

Internet: www.pedocs.de

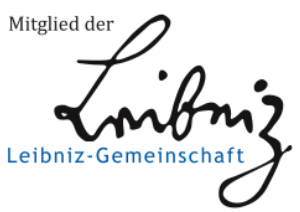




\title{
Renate Valtin
}

\section{Phonologische Bewusstheit: Ein kritischer Blick auf ein modisches Konstrukt}

\author{
Es scheint so, als hätten die Pädagogischen Psychologen mit der „phonologischen Bewusst- \\ heit" einen neuen „geistigen Muskel“ entdeckt, den es schon im Vorschulalter zu kräftigen \\ gilt. Ist er schlaff, handelt es sich um ein "Risikokind“, dem das Versagen im Lesen und \\ Schreiben droht. Die Botschaft heißt: Schon vor Schulbeginn sollen wir die phonologische \\ Bewusstheit diagnostizieren und "Defizite" durch ein gezieltes Training kompensieren - bei- \\ des mithilfe kostenpflichtiger kommerzieller Materialien. \\ Der Beitrag setzt sich in 6 Thesen kritisch mit diesem Konzept auseinander ${ }^{1}$.
}

\section{1. „Phonologische Bewusstheit“ ist linguistisch gesehen ein Wischi-Waschi- Konzept}

Bei der phonologischen Bewusstheit soll es darum gehen, die Lautstruktur der Sprache zu erfassen, wobei es unterschiedliche Definitionen gibt (vgl. Valtin 2010). Selbst Anhänger dieses Konzepts stellen fest, dass es sich „um ein Konglomerat ganz unterschiedlicher Teilfähigkeiten“ handelt, die "unterschiedlich komplex sind“ (Mayer 2011, S. 50).

Linguistisch macht ein derartiges Konzept keinen Sinn, denn es werden ganz verschiedene Einheiten (Wörter, Silben, Phoneme) und Operationen in einen Topf geworfen. Wörter sind eine grammatische Kategorie, und das Segmentieren von Einzelwörtern ist eine grammatische Operation, die nichts mit der phonologischen Ebene zu tun hat. Silben sind elementare, artikulatorische Einheiten des Sprechens. Phoneme sind abstrakte Einheiten (Sprachlaute mit bedeutungsunterscheidender Funktion), wobei Sprechlaute künstlich klassifiziert werden. Unterschiedliche Tests messen Unterschiedliches, und selbst ähnliche Aufgaben korrelieren nur schwach miteinander (s. die Analysen von Mayer 2011). Statt von phonologischer Bewusstheit zu reden, sollten also die spezifischen Teilfähigkeiten benannt werden.

\section{Die Forschung geht weitgehend theoriefrei vor}

Der Bezug zum Schriftspracherwerb wird nur behauptet bzw. durch Korrelationen belegt. Nun sagt eine Korrelation nichts aus über eine kausale Beziehung; sie kann auch auf gemeinsamer Varianz in einem weiteren Merkmal beruhen, z. B. IQ, Verbalintelligenz, Deutschkenntnisse, Wortschatzreichtum, Hörverstehen, Aufmerksamkeit und vorschulischer Lesefertigkeit. Zudem sind die herangezogenen Maße für Lesen und Rechtschreiben produktorien-

\footnotetext{
${ }^{1} 1$ Die Kürze dieses Beitrags erlaubt es nicht, in differenzierter Form alle Argumente aufzuführen und alle Studien zu zitieren. Wer an einer längeren Version dieses Beitrags mit einem ausführlichen Literaturverzeichnis interessiert ist, möge bitte schreiben an renate.valtin@rz.hu-berlin.de
} 
tiert und entsprechen nicht dem Erkenntnisstand zu Entwicklungsmodellen des Schriftspracherwerbs und qualitativen Fehleranalysen in Kompetenzmodellen der Orthographie. Dass das Erkennen von Silben, Wörtern und Sprachlauten psycholinguistisch unterschiedliche Leistungen sind, die unterschiedliche kognitions-, entwicklungs- und spracherwerbspsychologische Grundlagen haben, ist schon seit Jahrzehnten gut dokumentiert und wurde in der Beziehung zum Schriftspracherwerb theoretisch und empirisch untersucht sowie interdisziplinär verortet (u. a. Downing \& Valtin 1984). Diese Arbeiten werden aber von den Anhängern der phonologischen Bewusstheit nicht zur Kenntnis genommen. Fazit dieser Forschungsrichtung ist, dass Kinder bereits im Vorschulalter in der Lage sind, Wörter in Sprechsilben zu gliedern und spontan bzw. nach kurzer Übung Reime zu bilden. Das Begreifen von Wörtern als linguistischen Einheiten und die vollständige Phonemanalyse sind jedoch Ergebnis einer Auseinandersetzung mit der geschriebenen Sprache. Wenn Kinder im Vorschulalter also nicht wissen, was ein Wort ist, und ein Wort nicht in Sprachlaute zerlegen können, ist dies kein Defizit, sondern Ausdruck der Tatsache, dass sie noch nicht Lesen und Schreiben gelernt haben.

\section{Die Vorhersagekraft der phonologischen Bewusstheit ist bescheiden}

Zur Gültigkeit der These der phonologischen Bewusstheit werden immer wieder zwei Arten von Belegen angeführt: Korrelationen zwischen vorschulischer phonologischer Bewusstheit und Lesen bzw. Rechtschreiben sowie Effekte von vorschulischen und schulischen Trainings auf das Lese- und Rechtschreibleistung. Die Befunde sind höchst uneinheitlich (was bei einem derartigen Misch-Konzept auch zu erwarten ist). Die Korrelationen (s. dazu im Überblick Hatz \& Sachse, 2010) reichen von fehlender Signifikanz bis hin zu niedriger bzw. mittlerer Höhe (etwa in ähnlicher Höhe wie vorschulische Buchstabenkenntnis, Intelligenz oder Benennungsgeschwindigkeit), so dass sie sich nicht zur Individualdiagnose eignen.

Widersprüchlich sind auch die Befunde zu einem Transfereffekt von vorschulischen Trainings auf Leistungen im Lesen und Schreiben, wobei isoliert rein phonologische Trainings oder solche in Verbindung mit Buchstaben durchgeführt wurden. Sie reichen von keinerlei bis hin zu moderaten Effekten, die mal nur im Lesen, mal stärker im Rechtschreiben, mal nur im 2. Schuljahr, mal nur bei Mädchen auftraten. Zitiert wird immer wieder die Studie zur Wirksamkeit des Würzburger Trainings (Schneider, Roth \& Ennemoser, 2000), in der Kinder mit schwachen Ausgangsleistungen trainiert wurden. Die Ergebnisse dieser Studie lassen sich jedoch nur schwer interpretieren. Nicht nur, weil - aus ethischen, also gerechtfertigten Gründen - eine nicht trainierte Kontrollgruppe fehlt, sondern vor allem wegen der geringen prognostischen Gültigkeit des Bielefelder Screenings. Da, wie wir aus anderen Studien wissen, die Mehrzahl der Kinder mit einem diagnostizierten Risiko gar keine Schwierigkeiten im Lesen und Rechtschreiben entwickelt, lässt sich aus dem Design nicht ableiten, ob die „nachhaltigen Trainingserfolge“ nicht eher ein Beleg für die geringe Vorhersagegenauigkeit des Screeningtests darstellen. Wie auch immer, Schneider (2001, S. 91/92) schreibt dazu: Die Effektstärken sind „doch nicht derart überwältigend, dass man der phonologischen Be- 
wusstheit im Hinblick auf die Entwicklung von Schriftsprachkompetenzen einen absolut dominanten, kausalen Status zuschreiben muss".

Bei phonologischen Trainings im ersten Schuljahr zeigte sich keine Überlegenheit der geförderten gegenüber nicht geförderten Kindern (zuletzt Hatz \& Sachse, 2010). Verschiedene pädagogische Studien zeigen, dass durch traditionelle Lese-Lehrmethoden, die Kinder direkt zum Begreifen des alphabetischen Prinzips anleiten, auch die Phonembewusstheit von Kindern mit anfänglich schwachen Leistungen geübt wird. Ein vorschulisches isoliertes Training von Lautanalyse und -synthese, kombiniert mit einem Buchstabentraining, ist - so das Fazit in begrenztem Umfang möglich und zeigt auch in einigen, nicht jedoch in allen Studien, einen kleinen Effekt auf den Erfolg im Lesen- und Rechtschreibenlernen. Es ist also „machbar“.

Als Erziehungswissenschaftlerin halte ich jedoch nicht alles, was Psychologen für machbar erklären, für pädagogisch sinnvoll und wünschenswert. Die Frage ist, ob sich Aufwand und Nutzen des Einsatzes kommerzieller Programme lohnen, zumal die Trainings häufig linguistisch fehlerhaft sind (s. unten) und in einem guten Erstunterricht im Lesen und Rechtschreiben in den ersten Schulmonaten ohnehin Phonemanalyse und-synthese gefördert werden.

\section{Phonologische Bewusstheit spielt nur eine begrenzte Rolle beim Schrift- spracherwerb}

Der Prozess des Lesen- und Schreibenlernens lässt sich deuten als Erwerb von Fähigkeiten und Einsichten in Funktion und Aufbau der Schrift, wobei charakteristische Stufen oder Strategien unterschieden werden können. Während die Fähigkeit der Vergegenständlichung von Sprache eine notwendige Voraussetzung darstellt, handelt es sich bei der phonemischen Bewusstheit um eine wichtige Komponente (nicht eine Voraussetzung) des Erlernens und der Beherrschung des alphabetischen Codes unserer Schrift, die Sprachlaute durch Schriftzeichen sichtbar macht. Die Fähigkeiten der Phonemsynthese und -analyse sind jedoch nur von Bedeutung in den ersten Phasen des Schriftspracherwerbs: bis zum Erlesen und zur phonetischen Verschriftung. Phonemanalyse und -synthese allein verhelfen nicht zu orthographisch korrekten Schreibungen.

\section{Einige Übungen der phonologischen Trainings sind linguistisch fehlerhaft und didaktisch unsinnig}

Eine Durchsicht verschiedener Materialien ergibt, dass sie viele linguistische Fehler enthalten (z. B. die Verwechslung von Buchstaben und Lauten, die Nichtunterscheidung von Kurz- und Langvokalen). Einerseits sollen Kinder genau hören, andererseits werden ihnen aber bei den Vokalen nur der Langvokal, also der Buchstabenname, angeboten. Wie sollen sie aber im Wort Bild (gesprochen mit Kurzvokal) ein /I/ (gesprochen als Langvokal) „hören“? Wie sollen sie hören, dass alle Wörter in dem Satz Ohrwurm Olli organisiert originelle Opern mit dem 
gleichen Laut beginnen? (s. die Aufgabe im Würzburger Trainingsprogramm http://www.phonologischebewusstheit.de/spiele/merk.htm). Zudem werden nicht alle Kinder die hier verwendeten Vokabeln kennen.

Im TEPHOBE (Mayer, 2011, S. 33) müssen die Kinder das Kunststück fertig bringen, aus dem rückwärts gesprochenen „Quatschwort [saum]“ das Wort „Maus“ zu erkennen, nicht „Muas", da der Diphthong ignoriert wird. Im Würzburger Trainingsprogramm sollen Kinder zwölf häufig vorkommende Buchstaben und den Lautbezug lernen (Schneider et al. 2000). Da außerhalb eines geschriebenen Wortes Buchstaben keinen Sinn machen, wurde ein Vorgehen gewählt, bei dem jeder Buchstabe mit einem Sinn- oder Naturlaut verknüpft wird, z. B. „aaaa“ als Schmerzenslaut beim Zahnarzt und „ssss“ mit dem Summen von Bienen. Der Lesedidaktiker Bosch hat diese Vorgehensweise schon 1937 kritisiert, da es vielen Kindern beim Lesen eines Wortes schwer fällt, diese Verknüpfungen wieder zu vergessen; statt „im“ lasen sie dann „iiii kräht der Hahn, mmm brummt der Bär“. In der Grundschule sind diese synthetischen Verfahren schon vor Jahrzehnten zugunsten der analytisch-synthetischen Methode aufgegeben worden, weil sie Kinder nicht zum Verständnis der Funktion eines Buchstaben führen bzw. dies sogar erschweren. Warum sollten wir jetzt in der Vorschule Kindern diese unsinnige Methode zumuten? Übrigens weigerten sich in der Studie von Schneider et al. (2000) einige Kindergärtnerinnen, dieses Training durchzuführen.

\section{Die Fokussierung auf die phonologische Bewusstheit führt zur Vernachläs- sigung anderer wichtiger Bereiche des Schriftspracherwerbs}

Aus pädagogischer und sprachdidaktischer Sicht ist ein isoliertes Funktionstraining im Vorschulalter abzulehnen. Sinnvoll und empfehlenswert sind hingegen:

- eine umfassende Sprachförderung (Wortschatz, Grammatik, Erzählen, Rollen- und Phantasiespiele)

- spielerische Erfahrungen mit Schrift und Schriftsprache, die konzeptionelle Schriftlichkeit durch Vorlesen und den Umgang mit Büchern (und damit auch die Motivation zum Lesenlernen) fördern

- Anleitung zur Vergegenständlichung von Sprache durch spielerische Übungen (Zungenbrecher, Silbenklatschen, Reime bilden) und Lernen von Gedichten.

Kurzum: Im Kindergarten sollte den Kindern Neugierde und Lust auf das Lesen- und Schreibenlernen in der Schule vermittelt werden. 


\section{Literatur}

Bosch, B. (1937). Grundlagen des Erstleseunterrichts. Korrigierte Reprintausgabe (1984).

Frankfurt: Arbeitskreis Grundschule.

Downing, J. \& Valtin, R. (Eds.). (1984). Language awareness and learning to read. New York: Springer.

Hatz, H. \& Sachse, S. (2010). Prävention von Lese-Rechtschreibstörungen. Auswirkungen eines Trainings phonologischer Bewusstheit und eines Rechtschreibtrainings im ersten Schuljahr auf den Schriftspracherwerb. Zeitschrift für Entwicklungspsychologie und Pädagogische Psychologie, 42 (4), $226-240$.

Mayer, A. (2011). Test zur Erfassung der phonologischen Bewusstheit und der Benennungsgeschwindigkeit ( TEPHOBE), Manual. München: Max Reinhardt.

Schneider, W. (2001) Training der phonologischen Bewußtheit. In K. J. Klauer (Hrsg.), Handbuch kognitives Training (S. 69 - 95). Göttingen: Hogrefe.

Schneider, W., Roth, E. \& Ennemoser, M. (2000). Training phonological skills and letter knowledge in children at risk for dyslexia: a comparison of three kindergarten intervention programs. Journal of Educational Psychology, 92 (2), $284-295$.

Valtin, R. (2010). Phonologische Bewusstheit - eine notwendige Voraussetzung beim Lesenund Schreibenlernen? leseforum.ch. Online-Plattform für Literalität, 2. Zugriff am 27.7.2012. Verfügbar unter

https://www.leseforum.ch/sysModules/obxLeseforum/Artikel/426/2010_2_Valtin_PDF.pdf

Verfasserin: Prof. Dr. Renate Valtin, Allgemeine Grundschulpädagogik an der HumboldtUniversität zu Berlin (i. R.), E-Mail: renate.valtin@rz.hu-berlin.de. 